\title{
Stimmenhören, Farben schmecken - was ist eigentlich normal?
}

Es scheint ganz einfach: Wer Stimmen hört, die andere nicht wahrnehmen, leidet unter einem psychopathologischen Symptom („akustische Halluzination“). Therapeutisches Ziel: Die Stimmen im Kopf zum Verschwinden bringen. Der Spur der „Normalität“ folgend, endet hier bereits das Verständnis Nicht-Betroffener - die Konfrontation mit der Wahrhaftigkeit des Erlebten reicht nicht über eine banale Psycho-Diagnose hinaus. Doch wer sich - wenigstens vorübergehend - auf das Phänomen einlässt, begegnet auch sich selbst: Sei es in Form der Stimme des eigenen Gewissens, sei es in den Einflüsterungen politischer Verschwörungen, sei es in dem spirituellen Kontakt mit Gott oder hinsichtlich von künstlerischen Inspirationen (die ja bei Ärzten überdurchschnittlich häufig sind).

In überzeugender Weise hat das „Netzwerk Stimmenhören“ mit der Website www.stimmenhoeren.de die Komplexität des Phänomens aus Betroffenensicht eingefangen und dargestellt. Interaktive Elemente (Forum, Gästebuch), aber auch vielfältige Schilderungen von Betroffenen oder ausgesuchte Weblinks zu
Selbsthilfegruppen, Netzwerken oder Fachinformationen runden die Website ab. Resümee: Einfach und beispielhaft. Übrigens: Je nach Methodik zeigen epidemiologische Studien eine Häufigkeit von Stimmenhören über längere Zeit von mehr als $10 \%$.

\section{Synästhesie}

Nur wenig von der Psychiatrie vereinnahmt ist das nicht weniger erstaunliche Symptom der „Synästhesie“ - also der Kopplung verschiedener Wahrnehmungsbereiche bei nur einem Reiz („verschmolzene Wahrnehmung“). Synästhetiker können beispielsweise Geräusche nicht nur hören, sondern auch Formen und vor allem Farben dazu sehen (audition colorée). Wie viele Menschen solche Fähigkeiten (sic - nicht Symptom!) haben, ist unbekannt, da die individuelle Normalität der Synästhesie kaum auffällt. Klar ist nur, dass viele Künstler Synästhetiker waren oder sind. Die Zahl der Informations-Websites zum Thema ist erstaunlich hoch. Hier nur eine kleine Auswahl von Internetauftritten, die unterschiedliche Aspekte des Phänomens vorstellen:
- www.synaesthesie.info - eine virtuelle Ausstellung zum „lauten Staunen“ oder „zarten Hinhören“, wie es gerade gefällt.

-www.synaesthesieforum.de - eine Mailingliste mit gehaltvollen Add-Ons. Lassen Sie sich weder die Galerie mit den vielen Beispielen noch die Linkliste entgehen.

- www.sensequence.de - hier finden Sie Antworten auf Fragen wie „wie schmeckt der Montag?“, „welche Form hat das Jahr?“ oder „welche Tage sind am größten?".

Hinweis: Eine häufige Frage von NichtSynästhetikern ist - ganz anders als beim Stimmenhören: „Kann ich das auch erlernen?“

Rainer H. Bubenzer, Berlin

Quellen:

1 Thomas P, Bracken P, Leudar I. Hearing voices: A phenomenological-hermeneutic approach. Cognit Neuropsychiatry 2004; 9 (12): 13-23

2 Tien AY. Distributions of hallucinations in the population. Soc Psychiatry Psychiatr Epidemiol 1991; 26 (6): 287-292 